\title{
Synthetic Colors of Carbon Stars
}

\author{
WALTER WINDSTEIG, ERNST A. DORFI, \\ SUSANNE HÖFNER, JOSEF HRON, \\ and FRANZ KERSCHBAUM
}

Institut für Astronomie der Universität Wien, Vienna, Austria

We have calculated a number of synthetic colors and the spectral energy distributions of C-rich circumstellar envelopes of pulsating AGB stars. From the frequency-dependent radiative transfer calculations we obtain the paths within the IRAS two-color diagrams as well as the profiles of various $\mathrm{CO}$ lines during the pulsational cycle. These theoretical results are also compared to ground-based and IRAS observations.

This work is supported by the Fonds zur Förderung der wissenschaftlichen Forschung, projects S7305-AST and S7308-AST. 